\title{
Natural Log Transformed Observed Area Under the Curve Infinity
}

National Cancer Institute

\section{Source}

National Cancer Institute. Natural Log Transformed Observed Area Under the Curve

Infinity. NCI Thesaurus. Code C161413.

The natural log transformed area under the curve (AUC) extrapolated to infinity,

calculated using the observed value of the last non-zero concentration. 